\title{
Refractory Dedifferentiated Chordoma
}

National Cancer Institute

\section{Source}

National Cancer Institute. Refractory Dedifferentiated Chordoma. NCI Thesaurus. Code C162729.

A dedifferentiated chordoma that does not respond to treatment. 